\title{
Usefulness of Anorectal Manometry for Diagnosing Continence Problems After a Low Anterior Resection
}

\author{
Audrius Dulskas ${ }^{1}$, Narimantas E. Samalavicius ${ }^{1,2}$ \\ ${ }^{1}$ National Cancer Institute, Vilnius; ${ }^{2}$ Centre of Oncosurgery, National Cancer Institute, Clinic of Internal, Family Medicine and Oncology, \\ Faculty of Medicine, Vilnius, Lithuania
}

Purpose: For several decades, the low anterior resection (LAR) with total mesorectal excision (TME) has been the gold standard for treating patients with rectal cancer. Up to $90 \%$ of patients undergoing sphincter-preserving surgery will have changes in bowel habits, so-called 'anterior resection syndrome.' This study examined patients' continence after a LAR for the treatment of rectal cancer.

Methods: This prospective study was performed between September 2014 and August 2015 at the National Cancer Institute and included 30 patients who underwent anorectal manometry preoperatively and at 3 and 4 months after a LAR, but 10 were excluded from further evaluation for various reasons. Wexner score was recorded preoperatively and 4 months after LAR (1 month after ileostomy repair).

Results: Postoperatively, 70\% of patients complained of some degree of soiling (incontinence to liquid stool), and 30\% experienced urgent defecation. Four months after surgery, these symptoms had somewhat abated. The anal resting pressure and the maximum squeezing pressure did not change significantly. Rectal capacity and compliance were reduced in all patients. The majority of patients demonstrated manometric anorectal changes and clinical anorectal function disorders during the first 4 months after surgery. The Wexner scores and the manometric findings showed no correlation.

Conclusion: Many patients undergoing a LAR with TME for the treatment of rectal cancer experience some degree of incontinence postoperatively. Anorectal manometry may be used as an additional tool for evaluating problems with continence after a LAR. No correlation between the Wexner score and the manometric findings was observed.

\section{Keywords: Rectal cancer surgery; Manometry; Anterior resection syndrome}

\section{INTRODUCTION}

For several decades, the low anterior resection (LAR) has been the treatment of choice for patients with rectal cancer [1]. Postoperatively, many patients who undergo a LAR present with increased daily bowel movements, urgency for defecation, and a variable degree of incontinence. These symptoms that were just

Received: August 13, 2015 - Accepted: June 30, 2016

Correspondence to: Audrius Dulskas, M.D.

Center of Oncosurgery, National Cancer Institute, 1 Santariskiu Street,

Vilnius LT-08406, Lithuania

Tel: +370-67520094, Fax: +370-272-0164

E-mail: audrius.dulskas@gmail.com

(c) 2016 The Korean Society of Coloproctology

This is an open-access article distributed under the terms of the Creative Commons Attribution NonCommercial License (http://creativecommons.org/licenses/by-nc/4.0) which permits unrestricted noncommercial use, distribution, and reproduction in any medium, provided the original work is properly cited. described define the "anterior resection syndrome" [2]. Sometimes, urinary and sexual dysfunction may occur [3]. Anorectal manometry is an objective means of assessing the resistance to spontaneous defecation provided by the anorectal sphincter mechanism and the sensory capabilities of the rectum to provide a feeling of imminent defecation. The aim of the present study was to evaluate clinically and manometrically patients undergoing a low anterior rectal resection.

\section{METHODS}

This prospective study was performed between September 2014 and August 2015 at the National Cancer Institute. Thirty patients with a histologically proven adenocarcinoma of the middle or the lower third of rectum were included in the study. Preoperative diagnosis was proven by using rigid sigmoidoscopy with biopsies, digital examination, pelvic magnetic nuclear resonance tomogra- 
phy or endorectal ultrasound. Two patients who underwent abdominoperineal resection, and 5 patients who had preoperative short course radiotherapy were not further evaluated. Three were lost during follow-up. Of the remaining 20, 12 were males and 8 were females; their mean age was $61 \pm 8.3$ years. None of them had disseminated disease on preoperative work-up or exploratory laparotomy or a history of preoperative incontinence. We excluded patients who needed (neo)adjuvant radiotherapy to reduce both the number of variables and the bias in the data (Table 1).

\section{Surgical technique and assessment}

All patients underwent a 'standard' low anterior rectal resection with total mesorectal excision $[1,4]$. Briefly, surgery was performed under general anesthesia. The sigmoid colon and rectum was removed with high ligation of the inferior mesenteric artery. Autonomic nerves were preserved [5]. The descending colon was anastomosed side-to-end to the anal canal by using the double-stapling technique (CEEA stapling device [Ethicon, Cincinnati, OH, USA] with an outer diameter of $29 \mathrm{~mm}$ or $31 \mathrm{~mm}$ ). A diverting loop ileostomy was routinely performed at a site that had been marked previously by the stoma nurse. All the patients had negative mar-

Table 1. Clinical and demographic characteristics of 20 patients who underwent low anterior resection

\begin{tabular}{lr}
\hline Variable & No. (\%) \\
\hline Sex & \\
Male & $12(60.0)$ \\
Female & $8(40.0)$ \\
Age (yr) & $61 \pm 2.3$ \\
Stage, TNM & \\
I & $7(35.0)$ \\
II & $10(50.0)$ \\
III & $3(15.0)$ \\
Tumor localization & $8(40.0)$ \\
$\quad$ Lower third & $12(60.0)$ \\
$\quad$ Middle third & $3.4 \pm 2.5$ \\
Wexner score & \\
\hline
\end{tabular}

Values are presented as mean \pm standard deviation or number (\%). gins on subsequent histological examination. Anorectal function was assessed by using anorectal manometry preoperatively and at 3 months and 4 months after the first operation following closure of the stoma. The Wexner scores [6] of the patients were recorded preoperatively and 4 months from the first operation.

\section{Wexner score}

The Wexner score examines the frequency of three types of faecal incontinence (solid, liquid, and gas) and their consequences (pad wearing and lifestyle alteration). For each item, the 5 frequency options range from never (score 0 ) to always (meaning at least once per day; score 4). The total score is the sum of the item scores and ranges from 0 (perfect continence) to 20 (complete incontinence).

\section{Anorectal manometry}

The patient was asked to miss one meal prior to the study and to evacuate his or her rectum at least 2 hours before the measurement was taken. The patient was placed in the left lateral position; then, a perianal inspection and a digital rectal examination were performed, after which the patients was given instructions concerning the anorectal manometry examination. We used a Memphis Biomedica 8-channel water-perfused flexible catheter (Medicalpuls, Bologna, Italy) with side-holes for pressure recording, and the procedure was performed using a station pull-through technique. A computerized system (Polygram Lower GI, Synectics Medical, Stockholm, Sweden) was used for data acquisition. After calibration and exclusion of air from the system, the transducer was placed in the rectum and withdrawn at a rate of $5 \mathrm{~mm} / \mathrm{sec}$. The recording was started after a 5-minute period of stabilization. First, the mean basal pressure was recorded; then, the patient was asked to voluntarily squeeze his or her perianal muscles as hard as possible and to retain the contraction for as long as possible. The maximum squeeze pressure and the duration of maximum squeeze were, thus, recorded. A standard balloon-tipped catheter with transducer was then placed with the balloon $8 \mathrm{~cm}$ from the anus. The perception of rectal filling and the capacity of the neorectum were measured by placing the balloon with its lower edge $5 \mathrm{~cm}$ above the anal verge and subsequently slowly, in a stepwise manner, inflating the balloon with air in $20-\mathrm{mL}$ increments. The thresholds of the patient's first perception of rectal filling, urge to

Table 2. Results of preoperative and postoperative manometry in patients undergoing a low anterior resection

\begin{tabular}{|c|c|c|c|c|c|}
\hline Parameter & Normal values & Preoperatively & 3 Months postoperatively & 4 Months postoperatively & P-value \\
\hline Resting pressure (mmHg) & $40-70$ & $53 \pm 10.9$ & $35 \pm 6.7$ & $43 \pm 5.7$ & 0.123 \\
\hline Squeezing pressure $(\mathrm{mmHg})$ & $80-160$ & $120 \pm 12.8$ & $90 \pm 10.6$ & $100 \pm 8.9$ & 0.075 \\
\hline Volume to first sensation (mL) & $<50$ & $43 \pm 9.8$ & $26 \pm 8.2$ & $29 \pm 6.3$ & 0.030 \\
\hline Volume to first stool (mL) & $70-150$ & $125 \pm 10$ & $66 \pm 9.7$ & $98 \pm 11.2$ & 0.020 \\
\hline Maximal tolerable volume & $>160$ & $175 \pm 13.2$ & $117 \pm 11.2$ & $140 \pm 8.2$ & 0.045 \\
\hline
\end{tabular}

Values are presented as mean \pm standard deviation unless otherwise indicated. 
defecate, and maximal tolerable volume were assessed.

\section{Statistical analysis}

Data are presented as means \pm standard deviations. Pre- and postoperative serial values were compared by using the Student $t$-test. Significance was assumed for $\mathrm{P}<0.05$. The Bland-Altman method was applied to evaluate the agreement between anorectal manometry and the Wexner score [7]. That method calculates the mean difference between two methods of measurement and the $95 \%$ limits of agreement as the mean difference (1.96 of the standard deviation of the difference). The $95 \%$ limits are expected to include $95 \%$ of the differences between the 2 measurement methods. The plot of such data is commonly called a Bland-Altman plot, and the associated method is usually called the Bland-Altman method.

\section{RESULTS}

The differences in the sphincter actions preoperatively and at 1 and 4 months after surgery were not statistically significant, but the changes in the rectal capacity were (Table 2). Postoperatively, balloon manometry revealed an overall reduction in rectal capacity and compliance. Although the values tended to recover steadily, the values at 4 months after surgery still had not returned to the preoperative values. The volume to first sensation was lower after surgery and at 4 months after surgery still had not returned to its preoperative values $(\mathrm{P}<0.05)$, as was the volume to first stool $(\mathrm{P}<$ $0.05)$. The urgent volume was remarkably reduced $(\mathrm{P}<0.05)$, which continued for 4 months. The maximal tolerable volume also showed an obvious reduction postoperatively $(\mathrm{P}<0.05)$, which continued for 4 months. The rectoanal inhibitory reflex was absent during the entire study period in all patients.

The Wexner continence score showed a preoperative median score of 3.4 (range, $0-6 ; n=20$ ) and a postoperative median score of 6.3 (range, $2-8 ; n=20$ ). Postoperatively, 14 patients $(70 \%)$ complained of some degree of soiling (incontinence to liquid stool), and $6(30 \%)$ complained of urgency for defecation. Four months after surgery, only 2 patients complained of these symptoms. Postoperative defecation (either solid or liquid) was well-controlled. The median number of postoperative bowel movements was 3 per day (range, 1-7 per day).

The $95 \%$ limits of agreement in our study were from -13.723 to 29.274, the mean difference was 7.776 (95\% CI, 5.725-9.826). A comparison of the Wexner scores to the values obtained by using anorectal manometry for bowel dysfunction after the use of a LAR to treat patients with rectal cancer showed that the 2 methods could not be used interchangeably because observed differences within the limits of agreement are clinically important.

\section{DISCUSSION}

Our study showed impaired anorectal function following the use of a LAR to treat patients with rectal cancer. In addition, the rectal cancer itself had impaired this function as was seen in the preoperative Wexner scores and the values obtained by using anorectal manometry.

Many factors are reported to play roles in continence disorders. Following a LAR, the faecal capacity of the neo-rectum is decreased, and the patients lack a faecal "storage" reservoir, which ultimately leads to increased bowel movements [8]. Our study showed reductions in both rectal capacity and compliance postoperatively. Although they tended to recover gradually, but still not reached the preoperative values at fourth months after surgery. Our 4-month observation of anorectal function recovery may be too short because most studies find functional improvement at 1 or 2 years after surgery [8-12]. The preoperative rectal capacity of a patient with rectal cancer may be smaller than that of a healthy person due to the space occupied by the tumor, especially in the lower rectum. For resolution of postoperative functional disorders, several studies have reported the benefit of the colonic J-pouch for providing reservoir function $[9,10]$. However, despite the use of a reservoir, most patients continue to suffer from defecatory disorders. A prospective study by CamilleriBrennan and Steele [12] showed the evolution of quality of life before rectal surgery and 1 year postoperatively. The authors concluded that quality of life scores dropped significantly below baseline from 3 to 12 months postoperatively. Other authors have stated that the quality of life improves during the first year and reaches a steady state 1 to 2 years after surgery.

Continence is affected by injury to the anal sphincter. At least a few injury mechanisms have been reported in the literature. The internal sphincter is known to be injured during rectal resection, and both direct and indirect causes have been reported. Direct injury is due to the dilation of the anus that is necessary to introduce the stapling device [13]. An indirect cause is damage to the pelvic nerves $[8,14,15]$. The nerves can be damaged during the mobilization of the rectum or following surgery when inflammation and fibrosis take place. Up to $85 \%$ of the resting pressure is produced by tonic contraction of the internal anal sphincter, which is innervated by the previously mentioned nerves. When the nerves are injured during mobilization of the rectum, a significant decrease in resting pressure will be recorded on anorectal manometry. In stapled cases, a direct stretching injury to the internal sphincter may contribute in part to the fall in resting pressure. The anal squeezing pressure, which is generated by contraction of the external anal sphincter and the puborectalis muscle, did not show a significant reduction following surgery because the motor supply of the external anal sphincter is by the somatic nerves, which are not injured during the operation. We found impairments in both the resting and the squeezing pressures, but the differences were not statistically significant. The rectoanal inhibitory reflex was absent during the entire study period in all patients. This may be explained by a rupture of the intramural nerve network during transection of the rectum. Most authors report 
the same or similar results $[16,17]$.

On clinical evaluation with the Wexner scores, we found no statistically significant differences between the preoperative and the postoperative scores. Most likely, the worse preoperative results were caused by the cancer itself while the LAR caused the worse postoperative function. We found no correlation between the Wexner scores and the results obtained by using anorectal manometry. The Wexner scale assesses only three incontinence types; it does not take in to account bowel movement frequency or other symptoms of anterior resection syndrome. Incontinence symptoms can be partly compensated by proper function of the sphincter complex (mainly by external anal sphincter). This might be the reason for the disagreement between the Wexner scores and the results of anorectal manometry.

In conclusion, the impairment of continence after a LAR seems to be multifactorial, including diminished rectal capacity and compliance, as well as impaired internal anal sphincter tone. Most of the functional impairments had clinically and manometrically improved somewhat 4 months postoperation. In this study, we found that anorectal manometry might be used as an additional tool for monitoring continence problems after a LAR, but we found no correlation between the Wexner scores and the manometric findings.

\section{CONFLICT OF INTEREST}

No potential conflict of interest relevant to this article was reported.

\section{REFERENCES}

1. Heald RJ, Husband EM, Ryall RD. The mesorectum in rectal cancer surgery: the clue to pelvic recurrence? Br J Surg 1982;69:613-6.

2. Bryant CL, Lunniss PJ, Knowles CH, Thaha MA, Chan CL. Anterior resection syndrome. Lancet Oncol 2012;13:e403-8.

3. Lange MM, van de Velde CJ. Urinary and sexual dysfunction after rectal cancer treatment. Nat Rev Urol 2011;8:51-7.

4. Heald RJ. The 'Holy Plane' of rectal surgery. J R Soc Med 1988; 81:503-8.

5. Pocard M, Zinzindohoue F, Haab F, Caplin S, Parc R, Tiret E. A prospective study of sexual and urinary function before and after total mesorectal excision with autonomic nerve preservation for rectal cancer. Surgery 2002;131:368-72.

6. Jorge JM, Wexner SD. Etiology and management of fecal incontinence. Dis Colon Rectum 1993;36:77-97.

7. Bland JM, Altman DG. Statistical methods for assessing agreement between two methods of clinical measurement. Lancet 1986;1:307-10.

8. Kakodkar R, Gupta S, Nundy S. Low anterior resection with total mesorectal excision for rectal cancer: functional assessment and factors affecting outcome. Colorectal Dis 2006;8:650-6.

9. Emmertsen KJ, Chen TY, Laurberg S. Functional results after treatment for rectal cancer. J Coloproctol 2014;34:55-61.

10. Dehni N, Parc R, Church JM. Colonic J-pouch-anal anastomosis for rectal cancer. Dis Colon Rectum 2003;46:667-75.

11. Tsunoda A, Kamiyama G, Narita K, Watanabe M, Nakao K, Kusano M. Prospective randomized trial for determination of optimum size of side limb in low anterior resection with side-to-end anastomosis for rectal carcinoma. Dis Colon Rectum 2009;52: $1572-7$.

12. Camilleri-Brennan J, Steele RJ. Prospective analysis of quality of life and survival following mesorectal excision for rectal cancer. Br J Surg 2001;88:1617-22.

13. Vassilakis JS, Pechlivanides G, Zoras OJ, Vrachasotakis N, Chrysos E, Tzovaras G, et al. Anorectal function after low anterior resection of the rectum. Int J Colorectal Dis 1995;10:101-6.

14. Desnoo L, Faithfull S. A qualitative study of anterior resection syndrome: the experiences of cancer survivors who have undergone resection surgery. Eur J Cancer Care (Engl) 2006;15:244-51.

15. Lee WY, Takahashi T, Pappas T, Mantyh CR, Ludwig KA. Surgical autonomic denervation results in altered colonic motility: an explanation for low anterior resection syndrome? Surgery 2008;143: 778-83.

16. O'Riordain MG, Molloy RG, Gillen P, Horgan A, Kirwan WO. Rectoanal inhibitory reflex following low stapled anterior resection of the rectum. Dis Colon Rectum 1992;35:874-8.

17. Pedersen IK, Christiansen J, Hint K, Jensen P, Olsen J, Mortensen $P E$. Anorectal function after low anterior resection for carcinoma. Ann Surg 1986;204:133-5. 